\title{
Numerical behaviour of a new LES model with nonlinear viscosity
}

\author{
J. M. Rodrígueza, R. Taboada-Vázquez ${ }^{\mathrm{a}, *}$ \\ ${ }^{a}$ Departamento de Matemáticas, Universidade da Coruña, 15071 A Coruña, Spain
}

\begin{abstract}
The objective of this paper is to present a new Large Eddy Simulation (LES) model obtained by filtering a generalized version of the Navier-Stokes equations with nonlinear viscosity. This new model is a generalization of the model introduced in [J. M. Rodríguez, R. Taboada-Vázquez, A new LES model derived from generalized NavierStokes equations with nonlinear viscosity. Computers and Mathematics with Applications 73 (2017) 294-303].

The new LES model, in which viscosity has been substituted by a non linear function of the strain rate tensor, has been implemented and validated using FreeFem++ codes. The numerical predictions have been compared with analytical solutions of the Navier-Stokes equations and it was found that the present model provides a more accurate approximation of the exact solution than that obtained by Direct Numerical Simulation (DNS) of the Navier-Stokes equations, even using a coarser mesh.

The model has also been validated by studying the unsteady flow over a backwardfacing step. In this case, our numerical predictions have been compared with the experimental measurements reported by Armaly et al. and the numerical results obtained by Chacón and Lewandowski. The new model performs satisfactorily in predicting these flows too.
\end{abstract}

Keywords: Large Eddy Simulation, nonlinear viscosity, Backward Facing Step Flow test.

2010 MSC: 76D05, 76F65, 35Q35, 35Q30, 65M60.

\section{Introduction}

The Large Eddy Simulation (LES) approach is a tool for modelling unsteady turbulent flow and it is extremely useful for the prediction of complex flows in engineering applications such as channel flows, combustion, automotive, nuclear power engineering, etc. (see [15] or [22], for example). LES models are more accurate and of wider applicability than Reynolds-Averaged Navier-Stokes (RANS) models and less computationally demanding than the Direct Numerical Simulation (DNS) approach (see [1]).

\footnotetext{
${ }^{*}$ Corresponding author

Email addresses: jose.rodriguez.sei jo@udc.es (J. M. Rodríguez), raquel.taboada@udc.es (R. Taboada-Vázquez)
} 
In LES models, the important large scales are fully resolved on grid scales while the effects of the smaller subgrid-scales are modelled.

The LES approach is widely used to investigate the turbulence at high Reynolds numbers and to develop and assess new turbulence models, that is our objective.

The first step of LES method, according to Berselli et al. [6], is filtering the NavierStokes equations. The filter chosen in our previous work [25] was the following Gaussian filter :

$$
\bar{f}(t, \mathbf{x})=\int_{-\infty}^{\infty} \int_{\mathbb{R}^{3}} G(s-t, \mathbf{y}-\mathbf{x}) f(s, \mathbf{y}) \mathrm{d} \mathbf{y} \mathrm{d} s
$$

with

$$
G(s, \mathbf{y})=\frac{\gamma_{T}^{1 / 2} \gamma_{L}^{3 / 2}}{16 \pi^{2} \eta^{4}} \exp \left(-\frac{\gamma_{T} s^{2}+\gamma_{L}|\mathbf{y}|^{2}}{4 \eta^{2}}\right)
$$

where $\eta>0$ is a small parameter related to the size of the filter, and $\gamma_{T}>0, \gamma_{L}>0$ are parameters related to the shape of the filter.

Next step, following [6], is to introduce a closure model for subgrid-scale stress. One of the most popular models of this type is that proposed by Smagorinsky ([23]). This model is sometimes too dissipative, and it has other theoretical problems. In [25] we modelled this dissipation by introducing a nonlinear viscosity in the NavierStokes equations. By acting in such a way we introduced the dissipation predicted by Kolmogorov directly in our model and, when applying the filter, we closed the LES model by approximating the subgrid-scale stress tensor using Clark approximation (see [7] and [26]).

So, to deduce the new LES model, we applied the filter to these generalized NavierStokes equations with a nonlinear effective viscosity

$$
\begin{aligned}
& \rho_{0}\left(\frac{\partial \mathbf{u}}{\partial t}+(\nabla \mathbf{u}) \mathbf{u}\right)=\rho_{0} \mathbf{f}+\nabla \cdot \mathbf{T} \\
& \nabla \cdot \mathbf{u}=0
\end{aligned}
$$

where $\mathbf{u}$ is the velocity field, $\mathbf{f}$ is the acceleration due to external forces and the stress tensor $\mathbf{T}$ is given by

$$
\mathbf{T}=-p \mathbf{I}+2 \mu_{e}(|\mathbf{D}|) \mathbf{D}, \quad \mathbf{D}=\frac{1}{2}\left(\nabla \mathbf{u}+\nabla \mathbf{u}^{T}\right),
$$

where $p$ is the pressure and the effective viscosity $\mu_{e}$, depending on the norm of the strain rate tensor $\mathbf{D}$, was chosen as

$$
\mu_{e}(|\mathbf{D}|)=\mu_{0}\left(1+\lambda^{2}|\mathbf{D}|^{2}\right)^{1 / 2}
$$

where $\mu_{0}>0$ is the dynamic viscosity, $\lambda>0$, and we have considered the norm of $\mathbf{D}$ as follows:

$$
|\mathbf{D}|^{2}=\sum_{i, j=1}^{3} D_{i j} D_{i j}
$$

The parameter $\lambda$ was considered constant in [25] but in this article (see next section) we will obtain a more general model allowing $\lambda$ to depend on time and $\mathbf{x}$. 
The model thus obtained in [25] is as follows:

$$
\begin{aligned}
& \frac{\partial \overline{\mathbf{u}}}{\partial t}+(\nabla \overline{\mathbf{u}}) \overline{\mathbf{u}}+\frac{1}{\rho_{0}} \nabla \bar{p}=\overline{\mathbf{f}}+\nabla \cdot(\mathbf{S}-\tau), \\
& \nabla \cdot \overline{\mathbf{u}}=0,
\end{aligned}
$$

where $\tau$ (the so called subgrid-scale stress tensor) reads

$$
\begin{aligned}
\tau_{i j} & =\overline{u_{i} u_{j}}-\bar{u}_{i} \bar{u}_{j} \\
& =2\left[\frac{1}{\gamma_{T}} \frac{\partial \bar{u}_{i}}{\partial t} \frac{\partial \bar{u}_{j}}{\partial t}+\frac{1}{\gamma_{L}} \nabla \bar{u}_{i} \cdot \nabla \bar{u}_{j}\right] \eta^{2}+O\left(\eta^{4}\right),
\end{aligned}
$$

and $\mathbf{S}$ is given by

$$
\begin{aligned}
S_{i j} & =\frac{2}{\rho_{0}}\left(\overline{\mu_{e}(|\mathbf{D}|) \mathbf{D}}\right)_{i j} \\
& =2 \nu\left[\left(1+K_{1}\right)^{1 / 2}+\left(1+K_{1}\right)^{-1 / 2} K_{2} \eta^{2}-\frac{1}{4}\left(1+K_{1}\right)^{-3 / 2} K_{3} \eta^{2}\right] \bar{D}_{i j} \\
& +2 \nu\left(1+K_{1}\right)^{-1 / 2} K_{i j} \eta^{2}+O\left(\eta^{4}\right)
\end{aligned}
$$

The notation introduced is:

$$
\begin{aligned}
& \nu=\mu_{0} / \rho_{0}, \quad K_{1}=\lambda^{2}|\overline{\mathbf{D}}|^{2} \\
& K_{2}=\lambda^{2} \sum_{m, n=1}^{3}\left[\frac{1}{\gamma_{T}}\left(\frac{\partial \bar{D}_{m n}}{\partial t}\right)\left(\frac{\partial \bar{D}_{m n}}{\partial t}\right)+\frac{1}{\gamma_{L}} \nabla \bar{D}_{m n} \cdot \nabla \bar{D}_{m n}\right] \\
& K_{3}=\frac{1}{\gamma_{T}}\left(\frac{\partial K_{1}}{\partial t}\right)^{2}+\frac{1}{\gamma_{L}} \nabla K_{1} \cdot \nabla K_{1} \\
& K_{i j}=\frac{1}{\gamma_{T}} \frac{\partial K_{1}}{\partial t} \frac{\partial \bar{D}_{i j}}{\partial t}+\frac{1}{\gamma_{L}} \nabla K_{1} \cdot \nabla \bar{D}_{i j}
\end{aligned}
$$

So, a nonlinear viscosity was introduced in (6) as part of the model itself rather than using it as a procedure to close the subgrid-scale stress tensor in (9), where we have used the Clark approximation instead.

This new model thus obtained reminds us in some way of the dynamic procedure of Germano (see [14]).

\section{Derivation of a new model when lambda is not constant}

We are interested in investigating what happens when $\lambda$ in (6) is allowed to depend on the time and the spatial variables $(\lambda=\lambda(t, \mathbf{x}))$. If we now apply the filter (1)-(2) to equations (3)-(4), but with non-constant $\lambda$, we obtain again (8)-(10) but now $\mathbf{S}$ is given by

$$
\begin{aligned}
S_{i j} & =2 \nu\left[\left(1+\hat{K}_{1}\right)^{1 / 2}+\left(1+\hat{K}_{1}\right)^{-1 / 2} \hat{K}_{2} \eta^{2}-\frac{1}{4}\left(1+\hat{K}_{1}\right)^{-3 / 2} \hat{K}_{3} \eta^{2}\right. \\
& \left.+2 \bar{\lambda}\left(1+\hat{K}_{1}\right)^{-1 / 2} \hat{K}_{4} \eta^{2}\right] \bar{D}_{i j}+2 \nu\left(1+\hat{K}_{1}\right)^{-1 / 2} \hat{K}_{i j} \eta^{2}+O\left(\eta^{4}\right)
\end{aligned}
$$


where the notation used is:

$$
\begin{aligned}
& \nu=\mu_{0} / \rho_{0}, \quad \hat{K}_{1}=\bar{\lambda}^{2}|\overline{\mathbf{D}}|^{2} \\
& \hat{K}_{2}=\sum_{m, n=1}^{3}\left[\frac{1}{\gamma_{T}}\left(\frac{\partial\left(\bar{\lambda} \bar{D}_{m n}\right)}{\partial t}\right)\left(\frac{\partial\left(\bar{\lambda} \bar{D}_{m n}\right)}{\partial t}\right)+\frac{1}{\gamma_{L}} \nabla\left(\bar{\lambda} \bar{D}_{m n}\right) \cdot \nabla\left(\bar{\lambda} \bar{D}_{m n}\right)\right] \\
& \hat{K}_{3}=\frac{1}{\gamma_{T}}\left(\frac{\partial \hat{K}_{1}}{\partial t}\right)^{2}+\frac{1}{\gamma_{L}} \nabla \hat{K}_{1} \cdot \nabla \hat{K}_{1} \\
& \hat{K}_{4}=\sum_{m, n=1}^{3}\left[\frac{1}{\gamma_{T}} \frac{\partial \bar{\lambda}}{\partial t} \frac{\partial \bar{D}_{m n}}{\partial t}+\frac{1}{\gamma_{L}} \nabla \bar{\lambda} \cdot \nabla \bar{D}_{m n}\right] \bar{D}_{m n} \\
& \hat{K}_{i j}=\frac{1}{\gamma_{T}} \frac{\partial \hat{K}_{1}}{\partial t} \frac{\partial \bar{D}_{i j}}{\partial t}+\frac{1}{\gamma_{L}} \nabla \hat{K}_{1} \cdot \nabla \bar{D}_{i j}
\end{aligned}
$$

Comparing (11)-(15) with (16)-(21) we observe that the terms $\hat{K}_{i}(i=1,2,3), \hat{K}_{i j}$ are equal to the terms $K_{i}(i=1,2,3), K_{i j}$, where $\lambda$ is replaced by $\bar{\lambda}$ (that is, filtered $\lambda$ ), that is not constant now. There is also a new term $\hat{K}_{4}$ that does not appear in (11)-(15) because it is zero when $\bar{\lambda}$ is constant.

\section{Numerical results}

The numerical results presented in [25] correspond to a one dimensional version of the model (8)-(15). We compared the results obtained with analytical and numerical solutions of the Burgers equations, and we have seen the good numerical behaviour of the model deduced. Even for large discretization steps the filtered model provided quite good approximations of the exact solutions.

Now we want to study the numerical behaviour of the two dimensional model proposed in [25] ((8)-(15)) and the model we have just presented in the previous section ((8)-(10), (16)-(21)). With this aim, firstly, we have numerically solved both models and compared the results obtained with exact solutions. Then, turbulent flow over a backward-facing step has been studied. All the numerical simulations have been performed using FreeFem++ (see [16]) running in the Supercomputing Center of Galicia (CESGA), see [8]. 


\subsection{Numerical comparison with some analytical solutions.}

Let us introduce the following family of analytical solutions of the Navier-Stokes equations considering a rectangular domain of length $L$ and height $h,[0, L] \times[-h / 2, h / 2]$.

$$
\begin{aligned}
u_{1}(x, y) & =U_{\max } \frac{h^{2}-4 y^{2}}{h^{2}}+\sum_{i=1}^{2} d_{i} \cos \left(a_{i} x\right) \sin \left(b_{i} y\right) \\
u_{2}(x, y) & =\sum_{i=1}^{2} \frac{a_{i}}{b_{i}} d_{i} \sin \left(a_{i} x\right)\left(1-\cos \left(b_{i} y\right)\right) \\
p(x, y) & =-\mu\left(\frac{8 x}{h^{2}} U_{\max }+\sum_{i=1}^{2} \frac{a_{i}^{2}+b_{i}^{2}}{a_{i}} d_{i} \sin \left(a_{i} x\right) \sin \left(b_{i} y\right)\right) \\
& -\frac{\rho_{0}}{2}\left[\frac{h^{2}-4 y^{2}}{h^{2}} U_{\max }+\sum_{i=1}^{2} d_{i} \cos \left(a_{i} x\right) \sin \left(b_{i} y\right)\right]^{2} \\
& +\frac{8 \rho_{0} y}{h^{2}} U_{\max } \sum_{i=1}^{2} \frac{d_{i}}{b_{i}}\left(\cos \left(b_{i} y\right)-1\right) \cos \left(a_{i} x\right)
\end{aligned}
$$

where we have chosen

$$
\begin{aligned}
a_{i} & =\frac{2 \pi q_{i}}{L}, \quad q_{i} \in \mathbb{Z}, i=1,2 \\
b_{i} & =\frac{4 \pi k_{i}}{h}, \quad k_{i} \in \mathbb{Z}, i=1,2
\end{aligned}
$$

in such a way that (22)-(23) fulfil the following boundary conditions:

$$
\begin{aligned}
& u_{1}(0, y)=y_{1}(L, y)=U_{\max } \frac{h^{2}-4 y^{2}}{h^{2}}+\sum_{i=1}^{2} d_{i} \sin \left(b_{i} y\right) \\
& u_{1}(x,-h / 2)=u_{1}(x, h / 2)=0 \\
& u_{2}(0, y)=u_{2}(L, y)=u_{2}(x,-h / 2)=u_{2}(x, h / 2)=0
\end{aligned}
$$

To be sure that (22)-(25) is a solution of the Navier-Stokes equations (that is, a solution of the equations (3)-(4) with $\left.\mu_{e}=\mu_{0}\right), \mathbf{f}=\left(f_{1}, f_{2}\right)$ has been chosen in the appropriate way.

We have introduced the values of the kinematic viscosity and the density of water at $20^{\circ} \mathrm{C}$, that is, $\nu=10^{-6} \mathrm{~m}^{2} \mathrm{~s}^{-1}$ and $\rho=998.2 \mathrm{kgm}^{-3}$. These simulations have been performed at a Reynolds number of 10000 (turbulent regime).

All the results have been obtained with a time step $\Delta t=10^{-4}$. The test stop for the time-stepping procedure has been set to

$$
\frac{\left\|u_{1}^{n+1}-u_{1}^{n}\right\|_{\infty}}{\left\|u_{1}^{n+1}\right\|_{\infty}}<10^{-4}
$$

while the test stop for the fixed-point algorithm used to compute $\tau$ and $S$ in (10), (11) and (16) is more demanding since it stops when the relative error is less than $10^{-5}$. 
In the numerical examples of this paper, the variable $\lambda=\lambda(t, \mathbf{x})$ has been chosen in the following way: it is constant $\left(\lambda_{c}\right)$ in the inner part of the domain and it goes linearly to zero at the boundaries, with boundary layer thickness of three elements.

When we solve the different models, the pressure is computed except for a constant value, so to be able to compare the errors made when calculating the pressure using the different models, we introduce the normalized pressure

$$
p^{*}=\bar{p}-\frac{\int_{-h / 2}^{h / 2} \int_{0}^{L} \bar{p} d x d y}{L h}
$$

and we study the errors obtained approximating $p^{*}$ instead of $p$.

The results that follow have been obtained for these values of the coefficients that appear in (22)-(25):

$$
d_{1}=0.1, d_{2}=0.01, q_{1}=k_{1}=1, q_{2}=k_{2}=10
$$

considering that the domain dimensions are $L=1$ and $h=0.1$

Firstly we are going to compare the errors that we obtain when we try to approximate this exact solution by Direct Numerical Solution (DNS) of Navier-Stokes equations (in this case $\lambda=0$ ) and solving the new model ((8)-(10), (16)-(21)) with $\eta=0$ and different values of $\lambda$ with mesh step size $h / 75$. We have chosen this mesh step size because we are not able to achieve convergence in the case $\lambda=0$ (DNS of NavierStokes equations) with coarser meshes. Velocity and $p^{*}$ absolute errors are shown in table 1 (in $L^{\infty}$ norm) and in table 2 (in $H^{1}$ and $L^{2}$ norm).

\begin{tabular}{|c|c|c|c|}
\hline$\lambda_{c}$ & $\begin{array}{c}L^{\infty} \text { norm } \\
\text { error for } u_{1}\end{array}$ & $\begin{array}{c}L^{\infty} \text { norm } \\
\text { error for } u_{2}\end{array}$ & $\begin{array}{c}L^{\infty} \text { norm } \\
\text { error for } p^{*}\end{array}$ \\
\hline 0 (NS) & $3.57 e-2$ & $4.18 e-2$ & $3.44 e-3$ \\
\hline 0.1 & $9.77 e-3$ & $1.86 e-2$ & $2.17 e-3$ \\
\hline 0.5 & $5.41 e-3$ & $9.31 e-3$ & $1.31 e-3$ \\
\hline 1 & $5.48 e-3$ & $7.06 e-3$ & $9.45 e-4$ \\
\hline 2 & $8.68 e-3$ & $7.63 e-3$ & $1.32 e-3$ \\
\hline
\end{tabular}

Table 1: $L^{\infty}$ norm error bounds for Navier-Stokes $(\lambda=0)$ and different values of $\lambda_{c}$ with mesh step size $h / 75$

We can observe that the errors are smaller when we introduce $\lambda \neq 0$. For $\lambda_{c}=1$, for the first component of the velocity the errors are 6 times smaller in $L^{\infty}$ norm, 7 times smaller in $L^{2}$ norm and 20 times smaller in $H^{1}$ norm. The error for the second component of the velocity is also 6 times smaller in $L^{\infty}$ norm but 30 times smaller in $L^{2}$ and $H^{1}$ norm. The improvement is not so significant for the pressure. These tables also show how the accuracy of the approximations depends not only on the mesh step size, but also on the election of $\lambda$.

We have plotted in figures 1-2 and 4-5 both components of the velocity at $x=0.5$ and $y=0.01125$, respectively. In figures 3 and 6 we show $p^{*}$ (see (30)) at $x=0.5$ 


\begin{tabular}{|c|c|c|c|c|c|c|}
\hline$\lambda_{c}$ & $\begin{array}{c}H^{1} \text { norm } \\
\text { error for } u_{1}\end{array}$ & $\begin{array}{c}L^{2} \text { norm } \\
\text { error for } u_{1}\end{array}$ & $\begin{array}{c}H^{1} \text { norm } \\
\text { error for } u_{2}\end{array}$ & $\begin{array}{c}L^{2} \text { norm } \\
\text { error for } u_{2}\end{array}$ & $\begin{array}{c}H^{1} \text { norm } \\
\text { error for } p^{*}\end{array}$ & $\begin{array}{c}L^{2} \text { norm } \\
\text { error for } p^{*}\end{array}$ \\
\hline 0 (NS) & $4.48 e 0$ & $7.74 e-7$ & $1.06 e+1$ & $1.89 e-6$ & $7.39 e-3$ & $1.11 e-8$ \\
\hline 0.1 & $4.63 e-1$ & $7.69 e-8$ & $3.62 e 0$ & $6.01 e-7$ & $4.24 e-3$ & $4.50 e-8$ \\
\hline 0.5 & $1.59 e-1$ & $5.78 e-8$ & $8.12 e-1$ & $1.24 e-7$ & $1.50 e-3$ & $1.08 e-8$ \\
\hline 1 & $2.05 e-1$ & $1.09 e-7$ & $3.61 e-1$ & $5.40 e-8$ & $1.09 e-3$ & $4.54 e-9$ \\
\hline 2 & $7.28 e-1$ & $5.50 e-7$ & $1.7 e-1$ & $2.66 e-8$ & $6.93 e-4$ & $1.38 e-9$ \\
\hline
\end{tabular}

Table 2: $H^{1}$ and $L^{2}$ norm error bounds for Navier-Stokes $(\lambda=0)$ and different values of $\lambda_{c}$ with mesh step size $h / 75$

and $y=0.01125$ too. We have chosen the values of $x$ and $y$ where the solution presents more oscillations. In these figures, we can observe the exact solution, the approximation obtained by Direct Numerical Solution of Navier-Stokes equations and the approximations provided by the new LES model for $\lambda_{c}=0.1,0.5,1$ and 2 . As we could expect after seeing tables 1 and 2 the worst approximation is the one provided by DNS of Navier-Stokes equations.

In figures 1 and 4 we appreciate that oscillations appear when $\lambda=0$. These oscillations decrease with $\lambda_{c}=0.1$ and disappear when $\lambda_{c}$ is larger. The approximations with $\lambda_{c}=0.5$ and $\lambda_{c}=1$ are quite accurate. If we increase the value of $\lambda_{c}\left(\lambda_{c}=2\right.$ for example) we loose accuracy because we are introducing too much viscosity. 




Figure 1: $u_{1}$ component of the velocity at $x=0.5$ for $R e=10000$, with different values of $\lambda_{c}$ with mesh step size $h / 75$.

In the case of the second component of the velocity (figures 2 and 5) all the approximations present oscillations but their amplitude decreases when we introduce $\lambda_{c}>0$.

The approximations of the pressure are also better when we introduce $\lambda \neq 0$ than the approximation obtained by solving Navier-Stokes equations. 


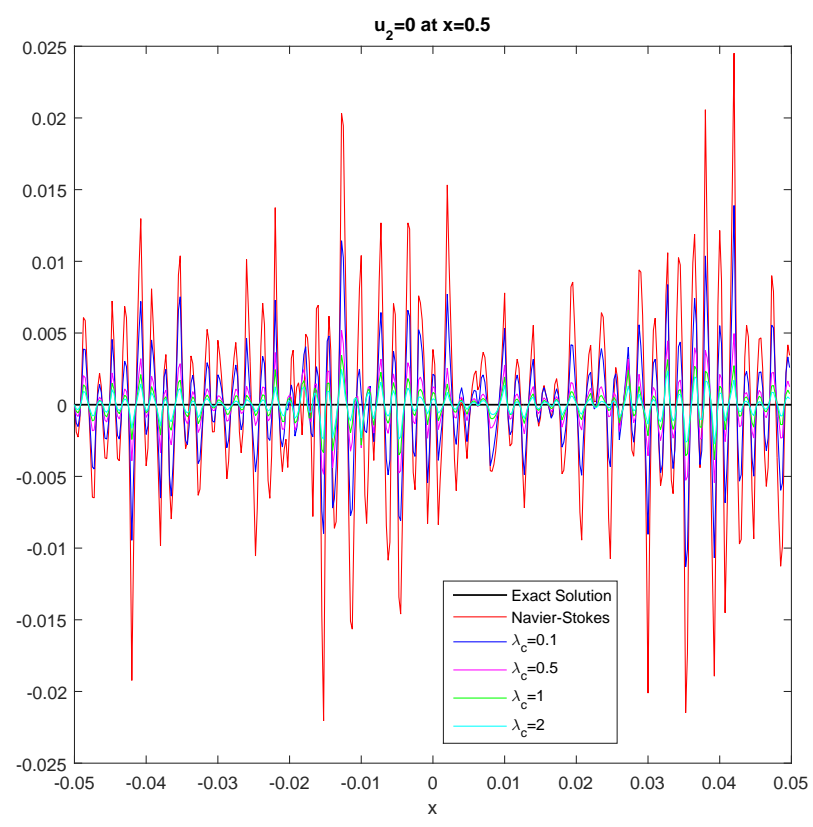

Figure 2: $u_{2}$ component of the velocity at $x=0.5$ for $R e=10000$, with different values of $\lambda_{c}$ with mesh step size $h / 75$.

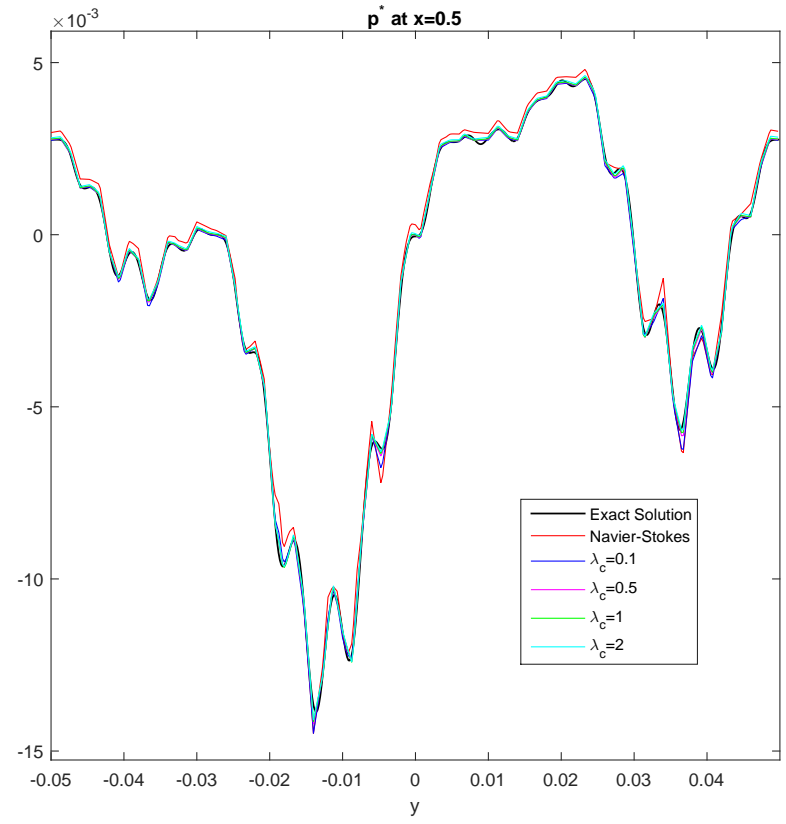

Figure 3: Pressure at $x=0.5$ for $R e=10000$, with different values of $\lambda_{c}$ with mesh step size $h / 75$. 


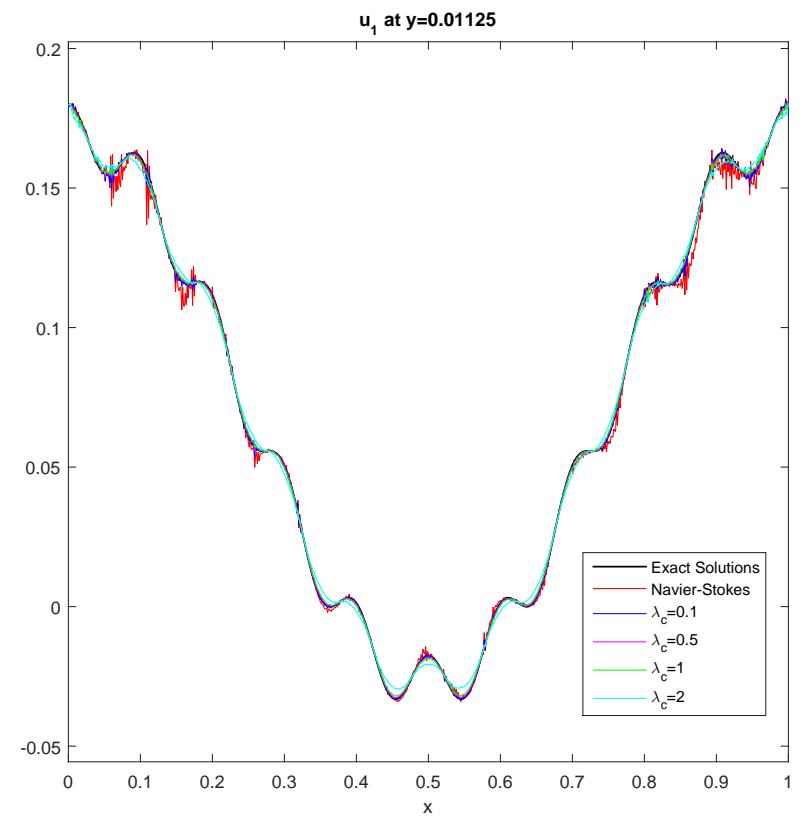

Figure 4: $u_{1}$ component of the velocity at $y=0.01125$ for $R e=10000$, with different values of $\lambda_{c}$ with mesh step size $h / 75$. 


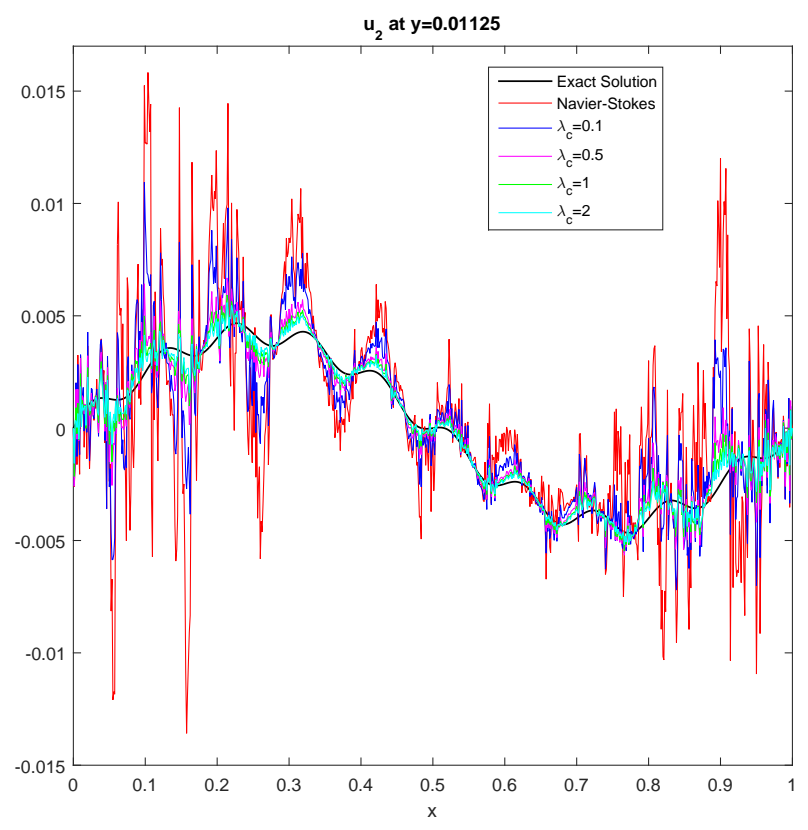

Figure 5: $u_{2}$ component of the velocity at $y=0.01125$ for $R e=10000$, with different values of $\lambda_{c}$ with mesh step size $h / 75$. 


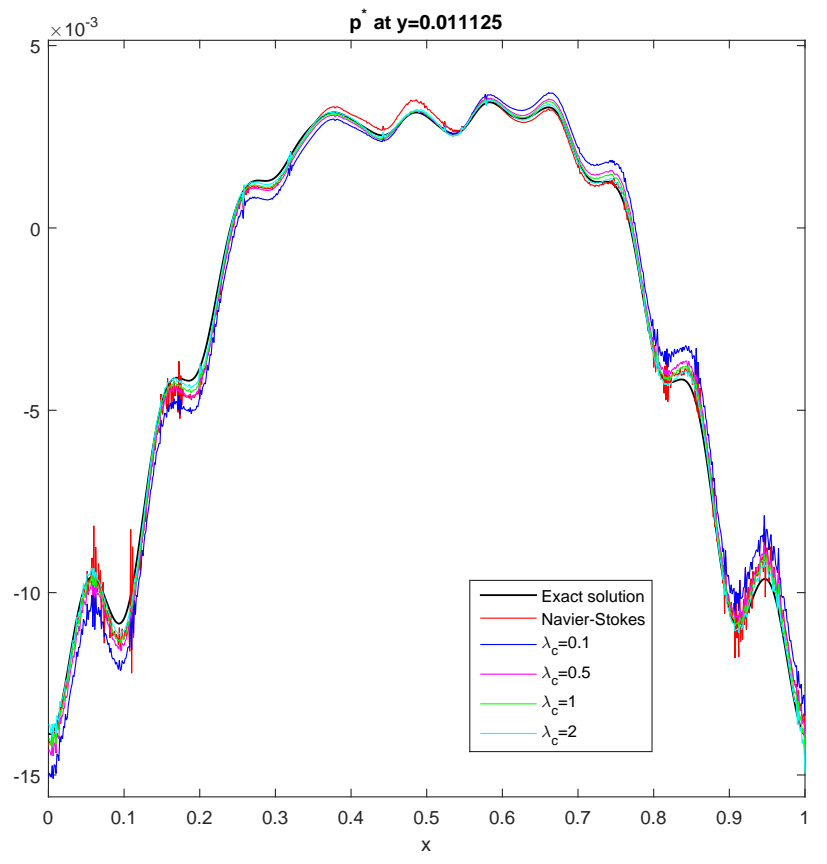

Figure 6: Pressure at $y=0.01125$ for $R e=10000$, with different values of $\lambda_{c}$ with mesh step size $h / 75$.

As we have already mentioned, the mesh step size cannot be larger than $h / 75$ to achieve convergence in the case $\lambda=0$ (DNS of Navier-Stokes equations). So, next we want to show (see table 3 ) that we are able to obtain more accurate solutions with the new model using much coarser meshes. In particular, table 3 shows that if we compute the new LES model with $\lambda_{c}=1$ and mesh step size $h / 55$ the velocity error is twice smaller than velocity error solving Navier-Stokes equations with mesh step size $h / 75$ in $L^{\infty}$ and $L^{2}$ norm and 10 times smaller in $H^{1}$ norm.

In tables 3-4 and figures 7-10, $n$ means that mesh step size is $h / n$.

\begin{tabular}{|c|c|c|c|c|c|c|}
\hline$\lambda_{c}$ & $n$ & $\begin{array}{c}L^{\infty} \text { norm } \\
\text { error for } u_{1}\end{array}$ & $\begin{array}{c}L^{\infty} \text { norm } \\
\text { error for } u_{2}\end{array}$ & $\begin{array}{c}L^{\infty} \text { norm } \\
\text { error for } p^{*}\end{array}$ & $\begin{array}{c}H^{1} \text { norm } \\
\text { error for } u_{1}\end{array}$ & $\begin{array}{c}L^{2} \text { norm } \\
\text { error for } u_{1}\end{array}$ \\
\hline 0 (NS) & 75 & $3.57 e-2$ & $4.18 e-2$ & $3.44 e-3$ & $4.48 e 0$ & $7.74 e-7$ \\
\hline 0 (NS) & 80 & $2.15 e-2$ & $2.57 e-2$ & $2.78 e-3$ & $1.37 e 0$ & $1.66 e-7$ \\
\hline 1 & 55 & $1.32 e-2$ & $1.48 e-2$ & $2.47 e-3$ & $4.66 e-1$ & $3.28 e-7$ \\
\hline 1 & 75 & $5.48 e-3$ & $7.06 e-3$ & $9.45 e-4$ & $2.05 e-1$ & $1.09 e-7$ \\
\hline
\end{tabular}

Table 3: Error bounds for Navier-Stokes $(\lambda=0)$ with $n=75$ and $\lambda_{c}=1$ variable with $n=55,75$

Even if we reduce the mesh step size $(h / 80)$, the errors obtained by using DNS are still significantly larger than those obtained with the new model with a mesh step size $h / 55$, see table 3 again. So, the results achieved with the new model are quite better using a mesh of less than the half of triangles and vertices (see table 4). 


\begin{tabular}{|c|c|c|}
\hline$n$ & Number of Triangles & Number of Vertices \\
\hline 80 & 147410 & 74586 \\
\hline 75 & 117778 & 59715 \\
\hline 55 & 64040 & 32623 \\
\hline
\end{tabular}

Table 4: Comparison of the number of triangles and vertices for different values of $n$

In figures 7-10 we can compare the velocity approximation provided by solving Navier-Stokes equations with mesh step size $h / 75$ with the velocity approximation obtained by computing the new model with mesh step size $h / 55$ at $x=0.5$ and $y=$ 0.01125 . We observe that the solution computed with the new model is more accurate although the mesh used is much coarser.

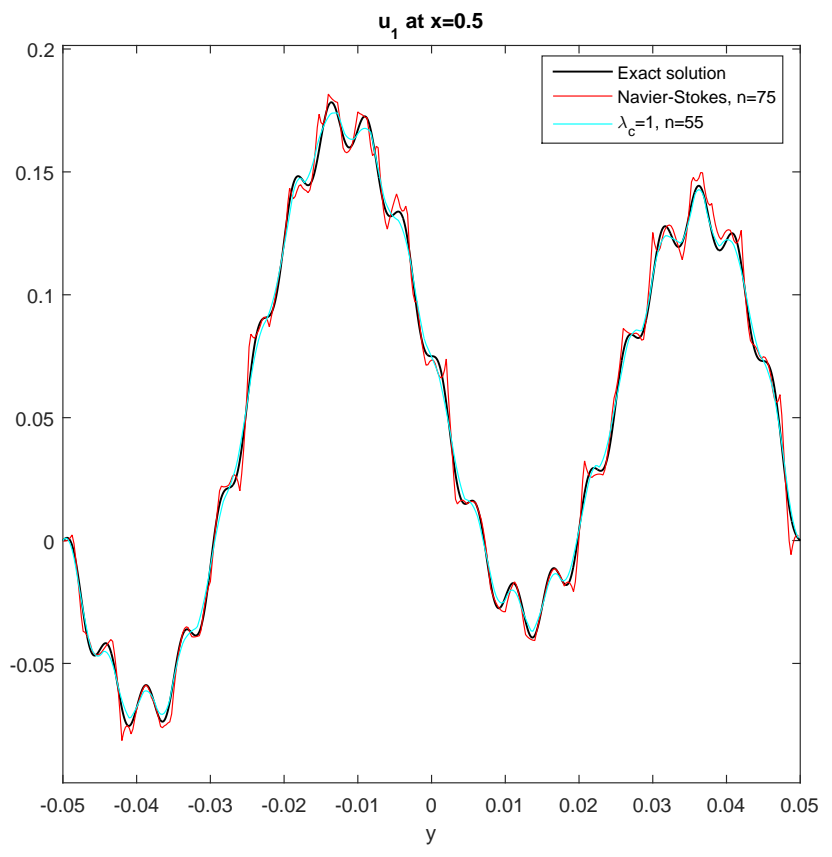

Figure 7: $u_{1}$ component of the velocity at $x=0.5$ for $R e=10000$, with $\lambda_{c}=1$ and without $\lambda$. 


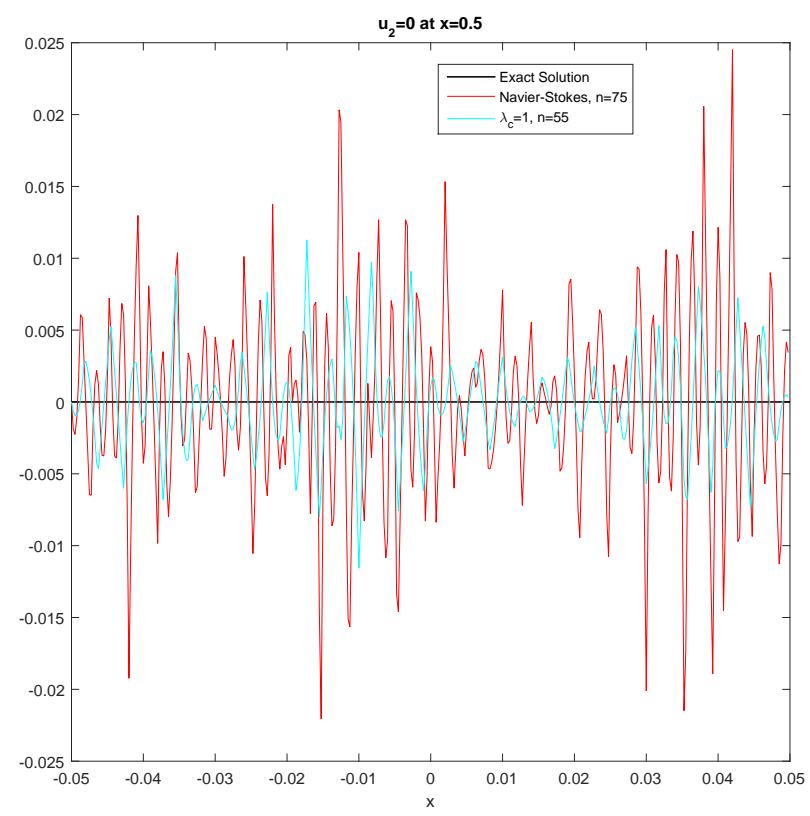

Figure 8: $u_{2}$ component of the velocity at $x=0.5$ for $R e=10000$, with $\lambda_{c}=1$ and without $\lambda$.

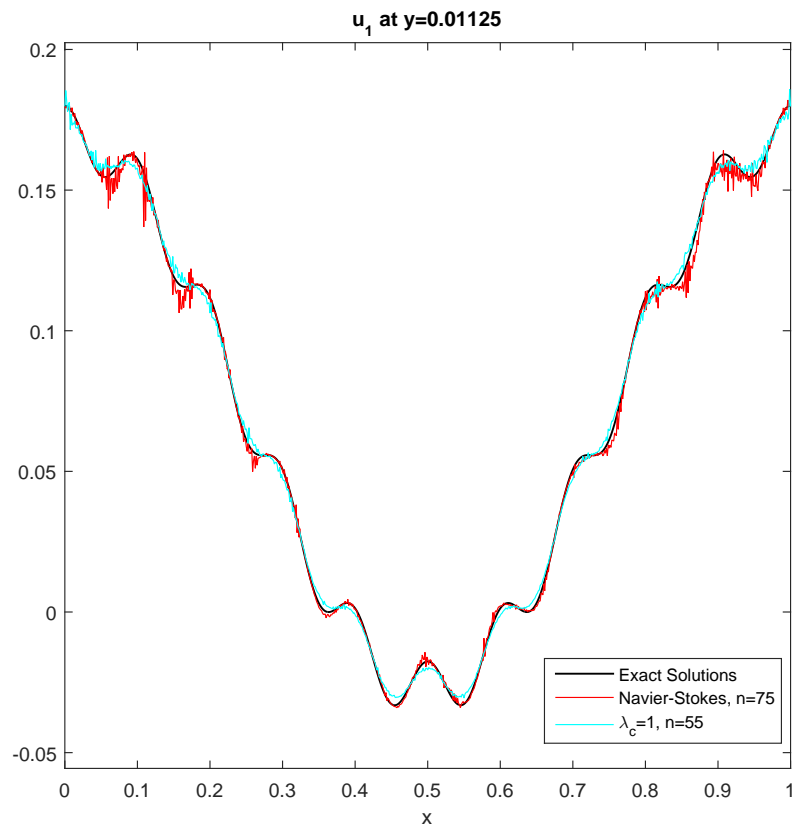

Figure 9: $u_{1}$ component of the velocity at $y=0.01125$ for $R e=10000$, with $\lambda_{c}=1$ and without $\lambda$. 


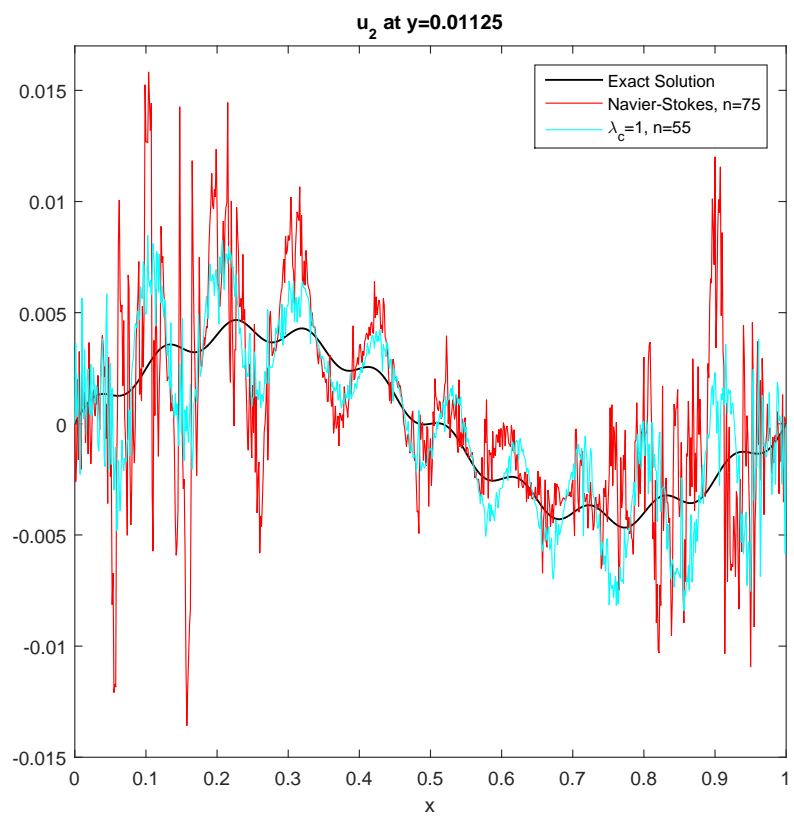

Figure 10: $u_{2}$ component of the velocity at $y=0.01125$ for $R e=10000$, with $\lambda_{c}=1$ and without $\lambda$.

The results obtained can be slightly improved by solving the filtered equations $(\eta \neq$ $0)$. Some of these improvements are shown in table 5, where the error bounds obtained by approximating (22)-(25) for the following values of the coefficients are given:

$$
d_{1}=0.1, d_{2}=0.01, q_{1}=k_{1}=1, q_{2}=k_{2}=2
$$

\begin{tabular}{|c|c|c|c|}
\hline$\lambda$ & $0(\mathrm{NS})$ & 2 & 2 \\
\hline$\eta$ & & 0 & $10^{-3}$ \\
\hline$L^{\infty}$ norm for $u_{1}$ & $1.91 e-3$ & $3.38 e-4$ & $2.31 e-4$ \\
\hline$H^{1}$ norm for $u_{1}$ & $2.67 e-3$ & $9.99 e-5$ & $5.60 e-5$ \\
\hline$L^{2}$ norm for $u_{1}$ & $1.04 e-9$ & $7.44 e-11$ & $3.81 e-11$ \\
\hline$L^{\infty}$ norm for $u_{2}$ & $2.39 e-3$ & $4.19 e-4$ & $3.06 e-4$ \\
\hline$H^{1}$ norm for $u_{2}$ & $1.16 e-2$ & $2.46 e-4$ & $1.37 e-4$ \\
\hline$L^{2}$ norm for $u_{2}$ & $4.02 e-9$ & $8.66 e-11$ & $4.81 e-11$ \\
\hline
\end{tabular}

Table 5: Error bounds for Navier-Stokes $(\lambda=0), \lambda=2$ with and without filtering for values $(32), n=50$

We observe that when $\eta=10^{-3}$ the velocity errors are nearly twice smaller than those obtained when $\eta=0$ in norm L-infinity and even smaller in norm $H^{1}$ and $L^{2}$.

The errors showed in table 6 were obtained with constant $\lambda$. We have observed that we obtain better results choosing a non-constant $\lambda$ than choosing a constant $\lambda$ when $n$ is large enough but for small values of $n$ the situation is the opposite. We think that this 
fact is due to our election of the boundary layer thickness, may be it is too large when $n$ is not large enough. A deeper study of how we should choose $\lambda$ should be carried out.

\subsection{Backward facing step flow}

The Backward Facing Step Flow test is widely used for turbulence validation and we want to examine the ability of our models to accurately compute the steady turbulent 2D backward step flow. The test consists in prescribing an inflow at a certain distance from the step, then the flow suddenly encounters the expansion of a wall, causing a flow separation, a large vortex is formed behind the step front. Armaly et. al. (see [2]) used a channel that had a height of $1.01 \mathrm{~cm}$ downstream the step and whose inlet was $0.52 \mathrm{~cm}(h)$ in height to conduct their study of this phenomenon.

So, we have computed this backward step flow by using both models ((8)-(15) with constant $\lambda$ and (8)-(10), (16)-(21) with variable $\lambda$ ) and then we have compared the results obtained with Armaly et al. experimental measures and the numerical results presented by Chacón and Lewandowski with different turbulence models (see [9]) for the backward facing step test case.

The definition of the Reynolds number which they used is given by

$$
R e=\frac{4 h V_{\max }}{3 \nu}
$$

where $V_{\max }$ is the maximum inlet velocity (the incoming flow is parabolic) and $\nu$ is the kinematic viscosity. Predictions of this flow, in a geometry equivalent to the case used for the experiments, were obtained by numerically solving the models proposed employing FreeFem++ again. The backward step length considered has been $l=4 h$ and the length of the computational domain 20 times the step height, in the same way as in [9].

When a variable $\lambda$ is chosen, it is constructed as in the previous section, that is, $\lambda=\lambda_{c}$ in the inner part of the domain and linearly decreasing to zero at the boundaries, with boundary layer thickness of three elements.

Several numerical simulations have been performed for different values of $\lambda$ (considered both constant and a function of $\mathbf{x}$ ) and different values of the Reynolds number. All the results presented here have been obtained with a mesh step size $h / 20$ and a time step 0.01 . The test stop for the time-stepping procedure has been set to

$$
\frac{\left\|u_{1}^{n+1}-u_{1}^{n}\right\|_{\infty}}{\left\|u_{1}^{n+1}\right\|_{\infty}}<10^{-5}
$$

In table 6 we present the computed (or measured) length of the main vortex formed. This length has been calculated by $X_{r} / h$ where $X_{r}$ is the reattachment length. 


\begin{tabular}{|c|c|c|c|c|c|}
\hline$R e$ & $\begin{array}{c}X_{r} / h \\
\text { measured [2] }\end{array}$ & $\begin{array}{c}X_{r} / h \\
\text { computed [9] }\end{array}$ & $\begin{array}{c}X_{r} / h \\
\lambda=0\end{array}$ & $\begin{array}{c}X_{r} / h \\
\text { constant } \lambda\end{array}$ & $\begin{array}{c}X_{r} / h \\
\text { variable } \lambda\end{array}$ \\
\hline 100 & 3 & $2.76-2.86$ & 2.83 & 2.85 & 2.85 \\
\hline 5000 & $\approx 6.9$ & $7.29-7.9$ & 14.85 & 6.94 & 8.72 \\
\hline 6000 & $\approx 6.3$ & $7.58-8.29$ & 14.89 & 6.34 & 9.02 \\
\hline 10000 & 8 & $8.02-8.85$ & - & 6.45 & 7.96 \\
\hline 20000 & 8 & & - & 6.83 & 7.60 \\
\hline
\end{tabular}

Table 6: Reattachment points $\left(X_{r} / h\right)$ for different Reynolds numbers

We can conclude that our models are able to compute the 2D backward step flow, reobtaining the reattachment lengths measured by Armaly et al. as accurately as Chacón and Lewandowski's models do for different Reynolds numbers. In the case of the laminar regime (for example, Reynolds number 100) Direct Numerical Solution of Navier-Stokes equations provides good results. For the values of the Reynolds number corresponding to the transition regime (for example, Reynolds number 5000 or 6000 ), the results obtained by DNS of Navier-Stokes equations are not good enough while the new models provide good approximations of the reattachment point. Finally, in the case of the turbulent regime (Reynolds number 10000 and 20000 considered), the scheme does not converge for $\lambda=0$ (Navier-Stokes equations) with the mesh step size we are considering. When we consider non-constant $\lambda$ the predictions of the secondary vortex are generally improved.

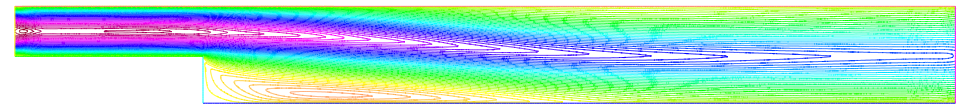

Figure 11: $u_{1}$ component of the velocity for $R e=10000$, non-constant $\lambda$

Figure11 shows the first component of the velocity map provided by the model considering non-constant $\lambda$, with $R e=10000$.

An additional recirculating-flow region was measured at the upper wall downstream of the expansion by Armaly et al. (see [2]). The authors report that this recirculation zone develops in the laminar range (for Reynolds numbers higher than 400) and it disappears above a Reynolds number of approximately $R e>6600$ (transition region). The new models proposed in this paper are able to obtain this recirculating-flow region (see figure 12 for $R e=5000$ for example). The beginning of the recirculation region found at the upper wall is upstream from the reattachment point of the primary recirculating flow region and its end is downstream from it, as Armaly et al. experimentally demonstrated. 


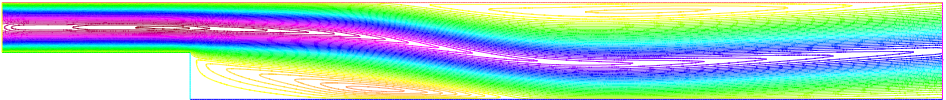

Figure 12: $u_{1}$ component of the velocity for $R e=5000$, constant $\lambda=0.07$

\section{Conclusions}

The LES model derived in [25] by applying a filter to the Navier-Stokes equations with a nonlinear effective viscosity and by approximating the subgrid-scale stress tensor using the Clark model, has been generalized by allowing the parameter $\lambda$ to be not constant.

We have performed some numerical simulations to test both new models. We have compared the results obtained with these new models (constant $\lambda$ and variable $\lambda, \eta=0$ and $\eta \neq 0$ ) with analytical and numerical solutions of the Navier-Stokes equations, and we have seen a good numerical behaviour of the models that we have derived. Even for coarser meshes the filtered models provide quite better approximations of the exact solutions than Navier-Stokes equations.

In the case of the Backward Facing Step Flow test, the new models are able to compute this flow, re-obtaining the reattachment lengths measured by Armaly et al. as accurately as Chacón and Lewandowski's models do for different Reynolds numbers.

\section{Acknowledgements}

This work has been partially supported by Ministerio de Economía y Competitividad (Spain) under grant MTM2016-78718-P with the participation of FEDER.

The numerical simulations have been performed using CESGA (Centro de Super Compuntación de Galicia)'s servers.

\section{References}

[1] C. D. Argyropoulos, N. C. Markatos, Recent advances on the numerical modelling of turbulent flows. Applied Mathematical Modelling 39 (2015), 693-732.

[2] B. F. Armaly, F. Durst, J. C.F. Pereira and B. Schöung, Experimental and theoretical investigation of backward-facing step flow. J. Fluid Mech. 127 (1983), 473-496.

[3] H. Beirão da Veiga, On the Ladyzhenskaya-Smagorinsky turbulence model of the Navier-Stokes equations in smooth domains. The regularity problem. J. Eur. Math. Soc. (JEMS) 11(1) (2009), 127-167.

[4] H. Beirão da Veiga, P. Kaplický, M. Rúžička, Regularity theorems, up to the boundary, for shear thickening flows. C. R. Math. Acad. Sci. Paris 348(9-10) (2010), 541-544. 
[5] L. C. Berselli, L. Diening, M. Rùžička, Existence of strong solutions for incompressible fluids with shear dependent viscosities. J. Math. Fluid Mech. 12(1) (2010), 101-132.

[6] L. C. Berselli, T. Iliescu, W. J. Layton, Mathematics of Large Eddy Simulation of Turbulent Flows. Springer, 2006.

[7] D. Carati, F. S. Winckelmans, H. Jeanmart, On the modelling of the subgridscale and filtered-scale stress tensors in large-eddy simulation. J. Fluid Mech. 441 (2001), 119-138.

[8] http://www.cesga.es/en/infraestructuras/computacion/FinisTerrae2

[9] T. Chacón, R. Lewandowski, Mathematical and Numerical Foundations of Turbulence Models and Applications. Birkhäuser, 2014.

[10] Y. M. Dakhoul, K. W. Bedford, Improved averaging method for turbulent flow simulation. Part I: Theoretical development and application to Burgers' transport equation. International Journal for Numerical Methods in Fluids 6 (1986), 49-64.

[11] Y. M. Dakhoul, K. W. Bedford, Improved averaging method for turbulent flow simulation. Part II: Calculations and verification. International Journal for $\mathrm{Nu}-$ merical Methods in Fluids 6 (1986), 65-82.

[12] L. Diening, M. Rủžička, J. Wolf, Existence of weak solutions for unsteady motions of generalized Newtonian fluids. Ann. Sc. Norm. Super. Pisa Cl. Sci. (5) 9(1) (2010), 1-46.

[13] J. Fröhlich, D. von Terzi, Hybrid LES/RANS methods for the simulation of turbulent flows. Progress in Aerospace Sciences 44(5) (2008), 349-377.

[14] M. Germano, U. Piomelli, P. Moin, W. H. Cabot, A dynamic subgrid-scale eddy viscosity model. Phys. Fluids A 3 (1991), 1760-1765.

[15] J.-L. Guermond, J. T. Oden, S. Prudhomme, Mathematical Perspectives on Large Eddy Simulation Models. J. Math. Fluid Mech. 6 (2004), 194-248.

[16] F. Hecht, New development in FreeFem++. J. Numer. Math. 20 (2012), no. 3-4, 251-265.

[17] B. E. Launder, B. I. Sharma, Application of the energy dissipation model of turbulence to the calculation of flow near a spinning disk. Lett. Heat Mass Transfer 1 (1974) 131-138.

[18] M. Lesieur, O. Métais, New trends in large-eddy simulations of turbulence. Annu. Rev. Fluid Mech. 28 (1996), 45-82.

[19] D. K. Lilly, A proposed modification of the Germano subgrid-scale closure method. Phys. Fluids A 4 (1992), 633-635. 
[20] J. Málek, J. Nečas, M. Rüžička, On the non-Newtonian incompressible fluids. Math. Models Methods Appl. Sci. 3(1) (1993), 35-63.

[21] W. Rodi, Turbulence models and their application in hydraulics, A state-of-the-art review. IAHR Monographs, third ed., CRC Press, 1993.

[22] P. Sagaut, Large Eddy Simulation for Incompressible Flows. Springer, 2006.

[23] J. Smagorinsky, General circulation experiments with the primitive equations, Mon. Weather Rev. 91 (1963), 99 - 164.

[24] P. R. Spalart, Detached-eddy simulation, Annu. Rev. Fluid Mech. 41 (2009), 181202.

[25] J. M. Rodríguez, R. Taboada-Vázquez, A new LES model derived from generalized Navier-Stokes equations with nonlinear viscosity. Computers and Mathematics with Applications 73 (2017) 294-303.

[26] B. Vreman, B. Geurts, H. Kuerten, Large-Eddy Simulation of the Temporal Mixing Layer Using the Clark Model. Theoret. Comput. Fluid Dynamics 8 (1996), 309-324.

[27] D. Wilcox, Turbulence Modelling for CFD. DCW Industries, Inc, 2006. 